\title{
Data mining analysis of terminal restriction fragment length polymorphism shows geographical differences in the human gut microbiota
}

\author{
AKIRA ANDOH ${ }^{1}$, TOSHIO KOBAYASHI ${ }^{2}$, HIROYUKI KUZUOKA $^{3}$, YASUO SUZUKI ${ }^{4}$, TOSHIYUKI MATSUI ${ }^{5}$, \\ SHIRO NAKAMURA $^{6}$, TAKAYUKI MATSUMOTO ${ }^{6}$, YOSHIHIDE FUJIYAMA $^{7}$ and TADAO BAMBA ${ }^{7}$ \\ ${ }^{1}$ Division of Mucosal Immunology, Graduate School, Shiga University of Medical Science, Otsu 520-2192; \\ ${ }^{2}$ Miyagi University, Sendai 982-0215; ${ }^{3}$ Research and Development Laboratories, EN Otsuka Pharmaceutical Co., Ltd., \\ Hanamaki, Iwate 025-0312; ${ }^{4}$ Department of Internal Medicine, Toho University Sakura Medical Center, Sakura 285-8741; \\ ${ }^{5}$ Department of Gastroenterology, Fukuoka University Chikushi Hospital, Chikushino, Fukuoka 818-8502; \\ ${ }^{6}$ Division of Lower Gastroenterology, Hyogo College of Medicine, Nishinomiya, Hyogo 663-850; \\ ${ }^{7}$ Department of Medicine, Shiga University of Medical Science, Otsu, Shiga 520-2192, Japan
}

Received April 25, 2013; Accepted May 14, 2013

DOI: $10.3892 /$ br. 2013.127

\begin{abstract}
Environmental factors are important for shaping the gut microbiota. In this study, terminal-restriction fragment length polymorphism (T-RFLP) analysis was performed, and data mining analysis was applied to investigate the geographical differences in the gut microbiota in Japan. A total of 121 healthy individuals living in four different districts (Shiga, Hyogo, Fukuoka and Chiba prefectures) in Japan were enrolled. Their gut microbiota profiles were evaluated by T-RFLP analysis, and data mining analysis using the Classification and Regression Tree (C\&RT) approach was performed. Data mining analysis provided a decision tree that clearly identified the various groups of subjects (nodes). Some nodes characterized the subjects from the four geographically distinct regions. Overall, 21 of the 35 subjects from the Hyogo Prefecture were mainly included in Node 21, 11 of the 16 subjects from the Shiga Prefecture were mainly included in Node 19, 37 of 40 subjects from the Chiba Prefecture were mainly included in Node 6 and 28 of 30 subjects from the Fukuoka Prefecture were included in Node 3. Only eight operational taxonomic units (OTUs) of the total 100 OTUs contributed to the characterization of the gut microbiota of the four geographically distinct districts in Japan. Geographical differences in the human gut microbiota were identified in Japan. Data mining analysis appears to be
\end{abstract}

Correspondence to: Professor Akira Andoh, Division of Mucosal Immunology, Graduate School, Shiga University of Medical Science, Seta Tukinowa, Otsu 520-2192, Japan

E-mail: andoh@belle.shiga-med.ac.jp

Key words: data mining, microbiota, terminal-restriction fragment length polymorphism one of the optimal tools for characterization of the human gut microbiota.

\section{Introduction}

The effect of gut microbiota on human health is recognized as a mutually beneficial interaction between human and indigenous microorganisms that contributes to normal physiology and immune homeostasis (1). De Filippo et al (2) recently demonstrated a difference in gut microbiota between European and African children, indicating that environmental factors such as diet, ethnicity, sanitation and hygiene are important for shaping the gut microbiota (3). Subsequently, it is likely that there are geographical differences in the gut microbiota within the Japanese population, since there are different lifestyles in different districts of the country. However, geographical differences in gut microbiota have not been previously investigated in Japan. Therefore, terminal-restriction fragment length polymorphism (T-RFLP) analyses of fecal samples from healthy individuals residing in different districts in Japan were performed, and data mining analysis was used to identify the geographical differences in gut microbiota.

\section{Materials and methods}

Healthy individuals. A total of 121 healthy individuals residing in four different districts in Japan [16 individuals from the Shiga Prefecture, 45 individuals from the Hyogo (and Osaka) Prefecture, 30 patients from the Fukuoka Prefecture and 40 individuals from the Chiba Prefecture] were enrolled in the present study (female/male, 52/69; mean age, 32.1 years of age). The location of each Prefecture in Japan is shown in Fig. 1. The Institutional Review Boards approved the study and written informed consent was obtained from each participant prior to enrolment. 
DNA extraction. The fecal samples were suspended in the buffer containing $4 \mathrm{M}$ guanidium thiocyanate, $100 \mathrm{mM}$ Tris- $\mathrm{HCl}(\mathrm{pH} 9.0)$ and $40 \mathrm{mM}$ EDTA and then beaten in the presence of zirconia beads using the FastPrep FP100A Instrument (MP Biomedicals, Irvine, CA, USA). The DNA was then extracted from the beads-treated suspension using the Magtration System 12GC and GC series Magtration-MagaZorb DNA Common kit 200N (Precision System Science, Chiba, Japan). The final concentration of the DNA sample was adjusted to $10 \mathrm{ng} / \mu \mathrm{l}$.

PCR amplification and T-RFLP analysis. The 16S rRNA gene was amplified from human fecal DNA using the 27 forward primer (5'-AGAGTTTGATCCTGGCTCAG-3') and the 1,492 reverse primer (5'-GGTTACCTTGTTACGACTT-3') $(4,5)$. The 5 '-ends of the forward primers were labeled with 6'-carboxyfluorescein (6-FAM), which was purchased from Applied Biosystems Japan (Tokyo, Japan). The PCR amplifications of the DNA samples (10 ng of each DNA) were performed according to a protocol previously described $(4,5)$. The amplified 16S rDNA genes were purified using MultiScreen PCR micro96 Plate (Merck Millipore, Tokyo, Japan) and dissolved in $40 \mu \mathrm{l}$ of distilled water.

The restriction enzymes were selected according to Matsumoto et al (4). The purified PCR products $(2 \mu \mathrm{l})$ were digested with 10 units of $H h a \mathrm{I}$ and $M s p \mathrm{I}$ at $37^{\circ} \mathrm{C}$ for $3 \mathrm{~h}$. The length of the T-RF fragments was determined using an ABI PRISM 310 Genetic Analyzer (Applied Biosystems, Tokyo, Japan) in GeneScan mode. Standard size markers, such as GS 2500 ROX (Applied Biosystems) were used. The fragment sizes were estimated using the local Southern method in the GeneScan 3.1 software (Applied Biosystems). Since the apparent size of identical T-RFs can vary over a range of 1-3 bp among different gels and/or lanes of the same gel, major T-RFs with a similar size of 1-3 bp were summarized as operational taxonomic units (OTUs). Hha1 and Msp1 digestion yielded 42 and 58 OTUs, respectively. The major T-RFs were identified by computer simulation, which was performed using a T-RFLP analysis program (6), a phylogenetic assignment database for the T-RFLP analysis of human colonic microbiota (4) and Microbiota Profiler (Infocom T-RFLP Database \& Analysis Software; Infocom Co., Tokyo, Japan).

Data mining. Data mining analysis was performed using SPSS Clementine 14 software (IBM Japan, Tokyo, Japan). A dividing system using the Classification and Regression Tree (C\&RT) approach, which is the most typical method for constructing decision trees, using the Gini coefficient (7) between geographic districts and operational taxonomic unit (OTU) data, was applied. The records were divided into two subsets in order that the records within each subset were more homogeneous than in the previous subset. The $\mathrm{C} \& \mathrm{RT}$ is flexible and allows unequal misclassification costs to be considered, unlike the other growing systems of data mining.

\section{Results and Discussion}

Data mining provided a decision tree (Fig. 2), which clearly identified the various groups of subjects (nodes). A decision

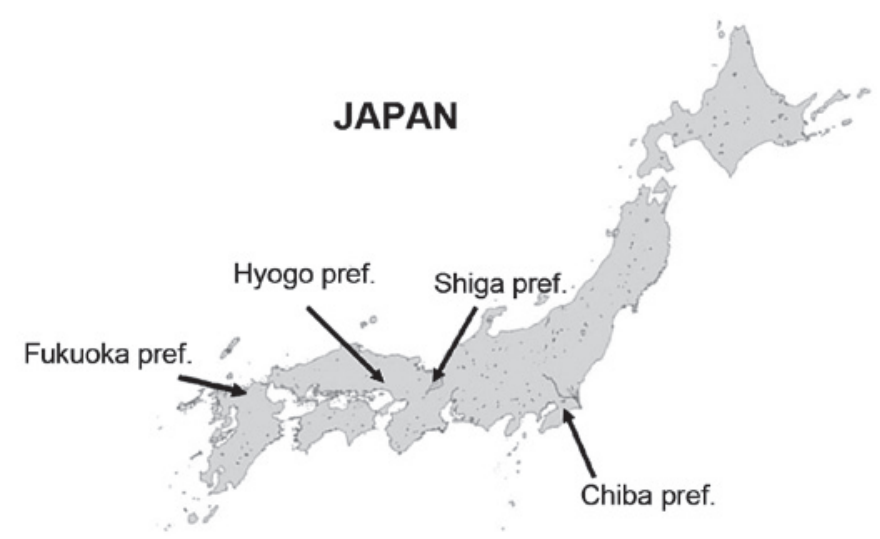

Figure 1. Locations of the four prefectures (Shiga, Hyogo, Fukuoka and Chiba) in Japan.

tree is a decision-supporting pathway that forms a tree-like graph. Each OTU was expressed as a restriction enzyme and RF length (base pair), e.g., HhaI 32 bp OTU was abbreviated as Hh32, and MspI 225 bp OTU was abbreviated as M225. Node-0, the left end of the decision tree, is known as the root node, which is the starting point for tree construction, while the nodes on the right indicate division of the various subject groups. As shown in Fig. 2, Node-0 was divided into Node- 1 and -2 by Hh32 with a cut-off value of 1.48 . This cut-off value of 1.48 of $\mathrm{Hh} 32$ was calculated from $\mathrm{Hh} 32$ data of all the subjects using the Gini coefficient with the C\&RT method. Similar steps were repeated for construction of the decision tree. The terminal node means the node that cannot be further divided as it has an impurity of 0 . The details of the decision tree and the pathway required to reach the terminal node clearly indicated the species and quantities of OTUs, which contributed to the division of the various subject groups.

Various nodes characterized the subjects from the four geographically distinct regions (Fig. 1). The subjects from the Hyogo Prefecture were mainly characterized by Node 21, comprising 21 of the 35 Hyogo subjects (60\%), and the subjects from the Shiga Prefecture were mainly characterized by Node 19 , comprising 11 of the 16 Shiga subjects (69\%). Similarly, the subjects from the Chiba Prefecture were characterized by Node 6, comprising 37 of the 40 Chiba subjects (93\%), and the subjects from the Fukuoka Prefecture were characterized by Nodes 3 (67\%), 12 and 24, which included 28 of the 30 Fukuoka subjects (total 93\%). These findings indicate the presence of geographical differences in the gut microbiota of healthy individuals in Japan.

A notable characteristic of data mining analysis is the use of a single selected OTU for each step of decision tree construction. In Fig. 2, only 8 of a total of 100 OTUs were used, with 2 OTUs (Hh32 and Hh93) being applied twice, meaning that the other 92 were not used to construct the tree shape. Thus, only 8 OTUs contribute to the characterization of gut microbiota of four geographically distinct districts in Japan.

We have previously reported the results of cluster analysis of the gut microbiota profiles of the same samples used in this study (8). However, we did not identify geographical differ- 


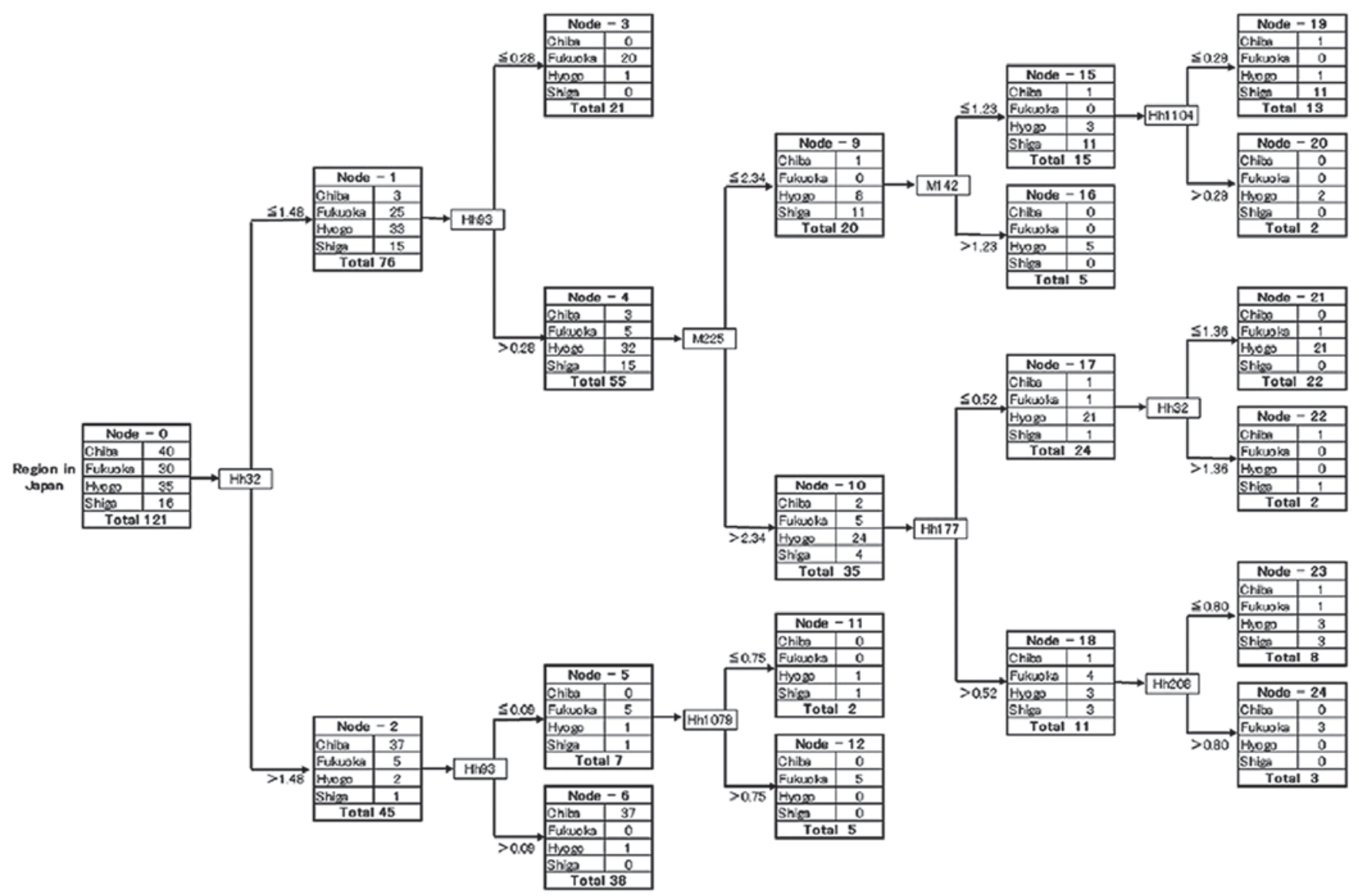

Figure 2. The decision tree was constructed using the Classification and Regression Tree (C\&RT) approach. Each operational taxonomic unit (OTU) is expressed as a restriction enzyme and restriction fragment length (base pair), e.g., HhaI 32 bp OTU is abbreviated as Hh32, and MspI 225 bp OTU is abbreviated as M225. The cu-toff value of each dividing OTU was calculated from OTU data of all the subjects, using Gini coefficient with the C\&RT method. Similar steps were repeated for construction of the decision tree. Node 0 (the left end of the decision tree) is known as the root node, which is the starting point for tree construction, and the terminal node is the node that cannot be further divided. Details of the decision tree and pathway required to reach the terminal node clearly indicate the species and quantities of OTUs, which contribute to division of the various subject groups.

ences using cluster analysis possibly due to two limitations of cluster analysis. The first is that the cluster analysis shows only some classified groups, but does not include obvious reasons for creating the groups. The second is that the obtained cluster lacks flexibility, thus a slight modification of the data affects cluster formation. On the other hand, data mining constructs a decision tree, which is a set rule that predicts target variables and creates the classification trees by repeatedly dividing the data. During this process, a tree branch is created, and each branch determines the classification criteria for the dividing data. Therefore, it explores the set of data and determines the variable that is predicted as the most significant of the predictor variables. Moreover, once the structure of the decision tree is constructed, as long as the basic concepts of the data were active, all of the subsequent new records can be run using the same data mining. The main difference between data mining and cluster analyses is the ability to handle data noise. Data mining skips characteristic noise and selects a series of related fields, but cluster processing respects all data without consideration of any numerical noise. Thus, using data mining analysis it was possible to demonstrate geographical differences in human gut microbiota in Japan.

In conclusion, to the best of our knowledge, this is the first report identifying geographical differences in human gut microbiota in Japan via construction of a decision tree and identification of 8 from a total of 100 OTUs. Given these results, the data mining method is considered to be one of the optimal tools for characterizing the human gut microbiota.

\section{Acknowledgements}

The authors appreciate the technical support of TechnoSuruga Laboratory Co., Ltd. (Sizuoka, Japan) and would like to express thanks.

\section{References}

1. Rautava S, Luoto R, Salminen S and Isolauri E: Microbial contact during pregnancy, intestinal colonization and human disease. Nat Rev Gastroenterol Hepatol 9: 565-576, 2012.

2. De Filippo C, Cavalieri D, Di Paola M, et al: Impact of diet in shaping gut microbiota revealed by a comparative study in children from Europe and rural Africa. Proc Natl Acad Sci USA 107: 14691-14696, 2010.

3. Kurokawa K, Itoh T, Kuwahara T, et al: Comparative metagenomics revealed commonly enriched gene sets in human gut microbiomes. DNA Res 14: 169-181, 2007.

4. Matsumoto M, Sakamoto M, Hayashi H and Benno Y: Novel phylogenetic assignment database for terminal-restriction fragment length polymorphism analysis of human colonic microbiota. J Microbiol Methods 61: 305-319, 2005. 
5. Sakamoto M, Takeuchi Y, Umeda M, Ishikawa I and Benno Y Application of terminal RFLP analysis to characterize oral bacterial flora in saliva of healthy subjects and patients with periodontitis. J Med Microbiol 52: 79-89, 2003.

6. Marsh TL, Saxman P, Cole J and Tiedje J: Terminal restriction fragment length polymorphism analysis program, a web-based research tool for microbial community analysis. Appl Environ Microbiol 66: 3616-3620, 2000.
7. Blair YI, McMahon AD and Macpherson LM: Comparison and relative utility of inequality measurements: as applied to Scotland's child dental health. PloS One 8: e58593, 2013.

8. Andoh A, Kuzuoka H, Tsujikawa T, et al: Multicenter analysis of fecal microbiota profiles in Japanese patients with Crohn's disease. J Gastroenterol 47: 1298-1307, 2012. 